\title{
Infinite Horizon Autonomous Systems with Unbounded Cost
}

\author{
A. Leizarowitz* \\ The Weizmann Institute of Science, Rehovot 76100 , Israel
}

Communicated by A. V. Balakrishnan

\begin{abstract}
We discuss control systems defined on an infinite horizon, where typically all the associated costs become unbounded as the time grows indefinitely. It is proved, under certain lower semicontinuity and controllability assumptions, that a linear time function can be subtracted from the cost, resulting in a modified cost, which is bounded on the infinite time interval. The cost evaluated over one sampling interval has a simple representation in terms of the initial and final states. Applying this representation we obtain an optimality result for control systems represented by ordinary differential equations whose cost integrand contains a discounting factor.
\end{abstract}

\section{Introduction}

In this work we analyze time invariant and periodic control systems, which operate on an infinite time horizon. Typically, such systems have optimal cost functionals which are unbounded as time tends to infinity. Three examples are displayed in the next section. It is not clear apriori how optimality should be defined when the cost tends to infinity. Several attempts were made in this direction; for instance, Gale [3] and von Weizsacker [6] developed the notion of overtaking, which was adopted by many authors.

The contribution of this work is in a level prior to the discussion of optimality. It is shown, under a controllability type condition, that a linear expression can be subtracted from the cost functional in a way that practically reduces the discussion to controls with bounded costs. The above-mentioned controllability condition is of the following nature: The set of admissible states of

\footnotetext{
*Current address: The Institute for Mathematics and Its Applications, University of Minnesota, Minneapolis, MN 55455.
} 
the system is a compact set, every member of which can be steered to any other one within a fixed finite time interval.

We develop a representation formula for the optimal cost which consists of splitting of the cost into three summands: a constant, a nonnegative term and a summand which depends only on the end-points of the trajectory and not on the trajectory itself. We shall demonstrate how such a representation is naturally suggested whenever the cost is convex.

The reduction to finite cost and the representation formula help in the analysis of the various optimality concepts. Some applications are discussed in the paper. In particular, we establish the existence of overtaking optimal solutions for control systems whose cost contains a discounting factor.

The paper is organized as follows. The framework is displayed in section 2 , along with three examples which motivate the abstract setting. In section 3 the reduction to finite cost is stated and proved while some comments appear in section 4. The representation formula is developed in section 5. Two applications of the abstract theory are given in sections 6 and 7 . One is concerned with the existence of an optimal overtaking solution where discounting factors are introduced into the cost-expressions. The other extends some early work of Bellman and Bucy [1] on continuous asymptotic control theory. In the final section we comment about an essential hypothesis of the model, namely the compactness assumption for the states; we show that in some cases the compactness can be derived from the structure of the problem.

\section{Motivation and Setting}

We first set forth the framework under which most of our results are developed. We find it convenient to work with discrete time models. This way we analyze the trajectories, which are in this context sequences in $R^{n}$, and which we call programs, rather than refer to the control action. The continuous case can be reduced to our framework as we demonstrate in the three examples which are displayed later on and which motivate our work.

The abstract model we analyze is summarized as follows:

Assumption I. Let $K$ be a compact set in the Euclidean $n$-dimensional space $R^{n}$, and let $v: K \times K \rightarrow R^{1}$ be bounded and lower semicontinuous (i.e. $v\left(\lim \left(x_{k}, y_{k}\right)\right)$ $\left.\leqslant \liminf v\left(x_{k}, y_{k}\right)\right)$.

The interpretation of the model is as follows. A control system is operating on an infinite time interval $[0, \infty)$. We choose a sampling time interval, say $[0, T]$. For any action that steers the state $x \in K$ at time $t=0$ to state $y \in K$ at time $t=T$ there is a cost associated. The value $v(x, y)$ appearing in Assumption $\mathrm{I}$ is the minimal cost possible. Any choice of control generates a trajectory, say $x(t)$, and we shall refer to $z_{k}=x(k T)$ as the program. If the control action is chosen in an optimal way on finite intervals the cost of the program $\left\{z_{k}\right\}$ at time $t=N T$ is $N-1$

$\sum_{i=0} v\left(z_{i}, z_{i+1}\right)$. We are interested, then, in the limit behaviour as $N \rightarrow \infty$ of such expressions.

Thus, in this paper we are interested in the programs $\left\{z_{k}\right\}$, which are sequences in $K$, rather than in the way to achieve them. A hidden assumption is 
that $v(x, y)$ is finitely defined on $K \times K$, namely a controllability type assumption. Generally this assumption is essential in order to establish our main results, as we demonstrate in section 4 by a counterexample. In certain convex problems it can be relaxed, namely, it suffices to assume the possibility of steering the initial state to some special state in a finite time (Brock and Haurie [2] establish their results under this assumption of controllability to a special point).

Another assumption is that $v$ is time-invariant; therefore the original control problem is, in general, either stationary or $T$-periodic. The lower semicontinuity of $v$ and its boundedness hold for many problems. The same is true for the compactness assumption, namely in many examples one can show that all the reasonable solutions occur in a prescribed compact set. We comment on this in section 8 .

Example I. A tracking problem. A linear time-invariant control system

$$
\dot{z}=A z+B u
$$

is given (here $z(t) \in R^{n}, u(t) \in R^{m}$ ) and suppose that it is controllable. The admissible control functions $u$ are the measurable functions on $[0, \infty)$ such that $\int_{0}^{T} u^{+}(t) u(t) \mathrm{d} t<\infty$ for every $t>0$, where the superscript + denotes transposition. A periodic trajectory $\Gamma(t)$ is prescribed and the purpose is to generate a trajectory $z(t)$ of $(2.1)$ which will be close to $\Gamma(t)$. (Controllability does not imply that $\Gamma(t)$ is an admissible trajectory.) If a quadratic criterion is adopted, then in applying the control $u(t)$ in the interval $[\tau, \tau+T]$, to which corresponds the trajectory $z(t)$ in that interval, the cost is given by

$$
c(u)=\int_{\tau}^{\tau+T}\left\{[z(t)-\Gamma(t)]^{+} Q[z(t)-\Gamma(t)]+u^{+} R u\right\} d t
$$

where the matrix $Q$ is positive semi-definite and $R$ is positive definite.

Typically, no matter how one chooses $u(t)$ on $[0, \infty)$, the cost on $[0, T]$ will diverge to infinity as $T \rightarrow \infty$.

To bring this problem under the framework described above we choose as a unit time the period of $\Gamma(t)$. We denote by $v(x, y)$ the minimal cost in (2.2) when $z(t)$ is subject to $z(0)=x$ and $z(T)=y$. Then $v$ is convex and continuous. As we show in section 8 , one can identify a compact set $K \subset R^{n}$ such that the requirements in assumption I are fulfilled.

Example II. Several authors studied the following problem. Given a continuous Lagrangian function $\mathscr{L}(z, \dot{z})$ consider the cost

$$
c_{T}(z)=\int_{0}^{T} \varphi(t) \mathscr{L}(z, \dot{z}) d t
$$

and study the existence of a function $z(t)$ for which the growth of $c_{T}(z)$ as $T \rightarrow \infty$ is minimal. In [4] Rockafellar deals with a problem originating in economics and $\varphi(t)$ there is an exponentially decreasing discounting factor. In [1] Bellman and Bucy consider such a problem with $\varphi(t) \equiv 1$.

We assume that the functions $z(t)$ and $\dot{z}(t)$ are measurable and are constrained to vary in compact sets in $R^{n}$. Such functions $(z(t), \dot{z}(t))$ are called an admissible pair. 
To bring this problem under our framework we denote by $v(x, y)$ the minimal value of $\int_{0}^{1} \mathscr{L}(z, \dot{z}) d t$ over all the admissible pairs $(z(t), \dot{z}(t))$ which satisfy $z(0)=x, z(1)=y$. In section 6 we follow this procedure in the case where $\varphi(t)$ is monotonically decreasing to zero.

Example III. This problem, like the previous one, has its roots in mathematical economics. As remarked by Brock and Haurie [2] the problems of optimal economic growth and optimal accumulation of capital by a profit maximizing firm are two examples of this problem in the context of economics. It can also be interpreted as a nonlinear tracking problem where the tracked trajectory is $\Gamma(t) \equiv 0$.

We consider a nonlinear system

$$
\dot{z}=f(z, u)
$$

with a cost

$$
c_{T}(u)=\int_{0}^{T} g(z, u) d t
$$

and an initial value $z(0)=z_{0}$. Both $f$ and $g$ are assumed to be continuous. The state function $z$ and the control function $u$ are subject to the constraints $z(t) \in K, u(t) \in \Omega$, where $K$ and $\Omega$ are compact sets in $R^{n}$ and $R^{m}$ respectively. The class of admissible controls is composed of all the measurable functions $u$ which satisfy $u(t) \in \Omega$ for all $t \geqslant 0$. A pair $(z, u)$ is admissible in $[0, T]$ if $u$ is an admissible control, $\dot{z}=f(z, u)$ and $z(t) \in K$ for all $0 \leqslant t \leqslant T$. We assume that given $(x, y) \in K \times K$ there is an admissible pair $(z(t), u(t))$ in $[0,1]$ which satisfy (2.4) together with $z(0)=x, z(1)=y$. The problem is to "minimize" (2.5) as $T \rightarrow \infty$. This problem fits into our framework in an obvious way.

Brock and Haurie [2] treated such problems with an additional convexity assumption. With convexity the controllability requirement may be relaxed (to that in [2]) as we note in section 4.

\section{The Reduction to Finite Costs}

We state now the main result, namely the existence of a reduction to finite costs. Recall that a program is a sequence in $K$. The proof will follow several propositions.

Theorem 3.1. There exist a constant $\mu$ and a constant $M>0$ such that:

${ }_{N}$ (1) For every program $\left\{z_{i}\right\}_{i=0}^{\infty}$ and every integer $N \geqslant 0$ the inequality $\sum_{i=0}^{N}\left[v\left(z_{i}, z_{i+1}\right)-\mu\right] \geqslant-M$ holds.

(2) For every program $\left\{z_{i}\right\}_{i=0}^{\infty}$ the sequence $\left\{\sum_{i=0}^{N}\left[v\left(z_{i}, z_{i+1}\right)-\mu\right]\right\}_{N=0}^{\infty}$ is either bounded or it diverges to infinity, and the set of all programs for which it is 
bounded is nonempty. Moreover, we can choose $M$ such that for every initial value $z_{0}$ there is a program $\left\{z_{i}^{*}\right\}_{i=0}^{\infty}$ with $z_{0}^{*}=z_{0}$ which satisfies

$$
\left|\sum_{i=0}^{N}\left[v\left(z_{i}^{*}, z_{i+1}^{*}\right)-\mu\right]\right| \leqslant M \text { for all } N \geqslant 0 .
$$

Just to indicate the usefulness of Theorem 3.1 we state two simple consequences of it. Let us introduce the following terminology: We shall call the sequence $\left\{\sum_{i=0}^{N} v\left(z_{i}, z_{i+1}\right)\right\}_{N=0}^{\infty}$ the cost flow of the program $\left\{z_{i}\right\}_{i=0}^{\infty}$. We shall also call the sequence $\left\{\sum_{i=0}^{N}\left[v\left(z_{i}, z_{i+1}\right)-\mu\right]\right\}_{N=0}^{\infty}$ the modified cost flow of the pro$\operatorname{gram}\left\{z_{i}\right\}_{i=0}^{\infty}$.

Corollary 3.2. The existence of a program whose cost flow diverges to infinity slower than any linear function, e.g. diverges like $\log N$, implies the existence of a program whose cost flow is bounded from above.

Proof. The reason is that the $\mu$ of such a system should be nonpositive, otherwise there would exist a program whose modified cost flow is not bounded below, in contradiction to (1) of Theorem 3.1. Since $\mu \leqslant 0$, the boundedness of the modified cost flow of the program $\left\{z_{i}^{*}\right\}_{i=0}$ (see (2) of Theorem 3.1) implies the boundedness of the cost flow of this program, and this proves our claim.

Corollary 3.3. If a program is such that at a certain time its modified cost exceeds $2 M$, then there is a program, with the same initial value, whose cost-flow is less than that of the former from that time on.

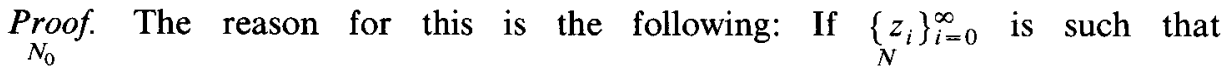
$\sum_{i=0}^{N_{0}}\left[v\left(z_{i}, z_{i+1}\right)-\mu\right]>2 M$, then by (1) of Theorem $3.1 \sum_{i=0}^{N}\left[v\left(z_{i}, z_{i+1}\right)-\mu\right]>M$ for all $N \geqslant N_{0}$, and by (2) of Theorem $3.1 \sum_{i=0}^{N}\left[v\left(z_{i}^{*}, z_{i+1}^{*}\right)-\mu\right]<M$ for all $N \geqslant 0$, where $z_{0}^{*}=z_{0}$. Therefore $\sum_{i=0}^{N} v\left(z_{i}^{*}, z_{i+1}^{*}\right)<\sum_{i=0}^{N} v\left(z_{i}, z_{i+1}\right)$, for all $N \geqslant N_{0}$, which is our assertion.

These two corollaries exhibit the fact that Theorem 3.1 is not just a growth-rate theorem, but it also says something about the costs at finite times. To prove Theorem 3.1 we need the following three lemmas. Denote by $\lambda(N)$ the minimal averaged cost over all periodic programs of period $N$; namely

$$
\lambda(N)=\min _{\substack{\left\{z_{i}\right\}_{i=0}^{N} \\ z_{0}=z_{N}}}\left\{\frac{1}{N} \sum_{i=0}^{N-1} v\left(z_{i}, z_{i+1}\right)\right\} .
$$


Let $\mu$ be defined as the infimal growth rate of cost flows over all programs, namely

$$
\mu=\inf _{\left\{z_{i}\right\}_{i=0}^{\infty}}\left[\liminf _{N \rightarrow \infty} \frac{1}{N} \sum_{i=0}^{N-1} v\left(z_{i}, z_{i+1}\right)\right]
$$

Remark. The quantity $\mu$ is a natural candidate to satisfy Theorem 3.1. In fact, if a quantity $\mu$ does satisfy Theorem 3.1, then it satisfies (3.2) too.

There is a close relation between the sequence $\{\lambda(N)\}$ in (3.1) and the constant $\mu$ defined in (3.2) described as follows:

Lemma 3.4. The following relation holds:

$$
\mu=\inf _{N \geqslant 1} \lambda(N) \text {. }
$$

Proof. On the one hand, it is easy to see that $\mu \leqslant \lambda(N)$ for every $N$, and therefore $\mu \leqslant \inf _{N \geqslant 1} \lambda(N)$. On the other hand, given an $\varepsilon>0$ there is a finite program with $N$ arbitrarily large such that $\frac{1}{N} \sum_{i=0}^{N-1} v\left(z_{i}, z_{i+1}\right)<\mu+\varepsilon$ and therefore $\frac{1}{N+1}\left[\sum_{i=0}^{N-1} v\left(z_{i}, z_{i+1}\right)+v\left(z_{N}, z_{0}\right)\right]<\mu+2 \varepsilon$, demonstrating that $\lambda(N+1)$ $<\mu+2 \varepsilon$, so that $\inf _{N \geqslant 1} \lambda(N) \leqslant \mu$.

In the sequel we shall use $\mu=\inf _{N \geqslant 1} \lambda(N)$ as the definition of $\mu$.

Lemma 3.5. The sequence $\lambda(N)$ converges to $\mu$.

Proof. Since $v(x, y)$ is bounded so is the sequence $\{\lambda(N)\}_{N=1}^{\infty}$ and let $\alpha$ $=\liminf _{N \rightarrow \infty} \lambda(N)$. We want to prove that $\lim \sup \lambda(N) \leqslant \alpha$ which will prove that $\{\lambda(N)\}_{N=1}^{\infty}$ is convergent. Given $\varepsilon>0$, there is an integer $N$ such that $\lambda(N)<\alpha$ $+\varepsilon$. Since every program which is periodic of period $N$ is also periodic of period $k \cdot N$ for every integer $k$, and as the averaged cost over a period of length $k N$ is the same as that over a period of length $N$ it follows from (3.1) that

$$
\lambda(k \cdot N) \leqslant \lambda(N) \text { for every } k \geqslant 1 \text { and } N \geqslant 1 \text {. }
$$

Further denote

$$
a=\sup _{(x, y) \in K \times K} v(x, y), \quad b=\min _{(x, y) \in K \times K} v(x, y) .
$$

Given any finite program $\left\{z_{i}\right\}_{i=0}^{N}$, replacing $z_{0}$ by $z_{0}^{\prime} \in K$ may increase the cost by at most $a-b$, since the difference between the costs is

$$
v\left(z_{0}, z_{1}\right)-v\left(z_{0}^{\prime}, z_{1}\right) \leqslant a-b
$$


Similarly the replacement of $z_{N}$ by any $z_{N}^{\prime} \in K$ may increase the cost by at most $(a-b)$. Let $N^{\prime}, N_{1}, N_{2}$ be integers such that $N^{\prime}=N_{1}+N_{2}$. Let $\left\{z_{i}\right\}_{i=0}^{N_{1}}$ be the program for which $\lambda\left(N_{1}\right)$ is attained and $\left\{s_{i}\right\}_{i=0}^{N_{2}}$ the one for which $\lambda\left(N_{2}\right)$ is attained. We replace $s_{0}$ and $s_{N_{2}}$ by $z_{0}=z_{N_{1}}$, identify $s_{0}$ with $z_{N_{1}}$ and form a finite program of length $N_{1}+N_{2}=N^{\prime}$

$$
\zeta_{i}= \begin{cases}z_{i} & 0 \leqslant i \leqslant N_{1} \\ s_{i-N_{1}} & N_{1}<i<N_{1}+N_{2} \\ z_{0} & i=N_{1}+N_{2}\end{cases}
$$

Using this program, for which $\zeta_{0}=\zeta_{N_{1}+N_{2}}$, we get $N^{\prime} \lambda\left(N^{\prime}\right) \leqslant N_{1} \lambda\left(N_{1}\right)+$ $N_{2} \lambda\left(N_{2}\right)+2(a-b)$. In particular take $N^{\prime}=k N+r$ where $1 \leqslant r<N$ then $N^{\prime} \lambda\left(N^{\prime}\right) \leqslant(k N) \lambda(k N)+r \lambda(r)+2(a-b)$, and divide by $N^{\prime}$ :

$$
\lambda\left(N^{\prime}\right) \leqslant \frac{k N}{k N+r} \lambda(k N)+\frac{r \lambda(r)+2(a-b)}{k N+r} .
$$

For $k$ large enough the second term in the last inequality is less than $\varepsilon$, and so by (3.3) and the way we chose $N$ we get

$$
\lambda\left(N^{\prime}\right) \leqslant \varepsilon(\alpha+\varepsilon) \frac{k N}{k N+r}<\alpha+3 \varepsilon
$$

and this for all large $k$ and every $1 \leqslant r<N$. Thus we conclude that $\limsup \lambda(k)$ $\leqslant \alpha$ since the preceding inequality is true for every $\varepsilon>0$.

$k \rightarrow \infty$

Had there been $N$ such that $\lambda(N)<\alpha$ then from (3.3): $\alpha=\liminf _{k \rightarrow \infty} \lambda(k)$ $\leqslant \liminf \lambda(i N) \leqslant \lambda(N)<\alpha$. So we get that $\alpha=\inf \lambda(N)$ and $\lim \lambda(N) \stackrel{k \rightarrow \infty}{=}$.

Lemma 3.5 gives a growth estimate to the cost of periodic programs. The next lemma gives a result which is finer than merely a growth rate.

Lemma 3.6. The following inequality holds:

$$
\limsup N[\lambda(N)-\mu]<\infty .
$$

$$
N \rightarrow \infty
$$

Proof. Denote by $\rho(N)$ the minimal averaged cost over all finite sequences of length $N$ (without any restrictions on the end values $z_{0}$ and $z_{N}$ ):

$$
\rho(N)=\min _{\left\{z_{i}\right\}_{i=0}^{N}}\left\{\frac{1}{N} \sum_{i=0}^{N-1} v\left(z_{i}, z_{i+1}\right)\right\} .
$$

We claim that $\rho(N) \leqslant \mu$ for every $N \geqslant 1$. Suppose to the contrary that $\rho(N)>\mu$ for some $N$. For every integer $k$

$$
\frac{1}{k N} \sum_{i=0}^{k N-1} v\left(z_{i}, z_{i+1}\right) \geqslant \frac{1}{k}(k \cdot \rho(N))=\rho(N),
$$


and this being true for every program $\left\{z_{i}\right\}$ implies $\rho(k N) \geqslant \rho(N)$. Also from the definitions of $\rho$ and $\lambda$ in (3.5) and (3.1), $\rho(k N) \leqslant \lambda(k N)$, so we get $\mu<\rho(N) \leqslant$ $\rho(k N) \leqslant \lambda(k N)$ for all $k \geqslant 1$. Letting $k \rightarrow \infty$ we get a contradiction since $\lambda(k N) \underset{k \rightarrow \infty}{\rightarrow} \mu$ by Lemma 3.5.

Recall the definition of $a$ and $b$ in (3.4). Let $N \geqslant 1$ be an integer and let $\left\{z_{i}\right\}_{i=0}^{N}$ be a finite program where $\rho(N)$ in (3.5) is attained. If we replace in this program $z_{N}$ by $z_{0}$ we get a periodic program of period $N$ whose cost in the interval $[0, N]$ does not exceed that of the former by more than $a-b$, so we get for every $N \geqslant 1$.

$$
N \cdot \lambda(N)-N \cdot \rho(N) \leqslant a-b .
$$

Combining this with $\rho(N) \leqslant \mu$ leads to

$$
\limsup _{N \rightarrow \infty} N[\lambda(N)-\mu] \leqslant \limsup _{N \rightarrow \infty} N[\lambda(N)-\rho(N)] \leqslant a-b .
$$

Before proving Theorem 3.1 let us introduce two abbreviating notations. We shall denote a program $\left\{z_{i}\right\}_{i=0}^{\infty}$ by a bold face letter $\mathbf{z}$, and for $N \geqslant 1$

$$
m_{N}(\mathbf{z})=\sum_{i=0}^{N-1}\left[v\left(z_{i}, z_{i+1}\right)-\mu\right]
$$

will be called the modified cost of $\mathbf{z}$.

Note that the modified cost of a finite periodic program is nonnegative, since from $z_{0}=z_{N}$ and $\mu=\inf \lambda(N)$ it follows that $\frac{1}{N} \sum_{i=0}^{N-1} v\left(z_{i}, z_{i+1}\right) \geqslant \mu$ and therefore $m_{N}(\mathbf{z}) \geqslant 0$.

Proof of Theorem 3.1. We shall prove first the claim in (1), namely that for every program $\mathbf{z}$ and every $N \geqslant 1$ the inequality $m_{N}(\mathbf{z}) \geqslant-(a-b)$ holds (recall (3.4)). Given $\mathbf{z}$ and $N$ we replace $z_{N}$ by $z_{0}$ and thus reduce the modified cost on the interval $[0, N]$ by no more than $a-b$. After the replacement, we get a periodic program, whose modified cost is nonnegative so we get $m_{N}(\mathbf{z}) \geqslant-(a-b)$ as claimed. Put formally we have

$$
\begin{aligned}
m_{N}(\mathbf{z})= & \left\{\sum_{i=0}^{N-2}\left[v\left(z_{i}, z_{i+1}\right)-\mu\right]+\left[v\left(z_{N-1}, z_{0}\right)-\mu\right]\right\} \\
& +\left[v\left(z_{N-1}, z_{N}\right)-v\left(z_{N-1}, z_{0}\right)\right]
\end{aligned}
$$

where the first term is nonnegative, while the second exceeds $-(a-b)$.

We prove now the assertion made in (2). We only have to prove the existence of a program $\mathbf{z}$ for which $\left\{m_{N}(\mathbf{z})\right\}_{N=1}^{\infty}$ is bounded, for every initial value, and that the bound is independent of the initial value. (It follows from (1) that if $\left\{m_{N}(\mathbf{z})\right\}_{N=1}^{\infty}$ is not bounded then it diverges to infinity.) Using Lemma 3.6 we choose $\gamma>0$ so that for all $N \geqslant 1$ we have

$$
N[\lambda(N)-\mu]<\gamma .
$$


Let $\left\{z_{0}^{N}, \ldots, z_{N}^{N}\right\}$ be chosen so that $z_{0}^{N}=z_{N}^{N}$ and $\lambda(N)=\frac{1}{N} \sum_{i=0}^{N-1} v\left(z_{i}^{N}, z_{i+1}^{N}\right)$. By (3.7) we get $\sum_{i=0}^{N-1}\left[v\left(z_{i}^{N}, z_{i+1}^{N}\right)-\mu\right]<\gamma$ for all $N \geqslant 1$. We claim that if $l \geqslant N \geqslant 1$
then

$$
\sum_{i=0}^{N-1}\left[v\left(z_{i}^{l}, z_{i+1}^{l}\right)-\mu\right]<\gamma+a-b
$$

namely, the modified cost of the finite programs $\left\{z_{0}^{l}, \ldots, z_{l}^{l}\right\}$ computed on the interval of length $N$ has a bound, uniform on $1 \leqslant N \leqslant l$. Let us assume the converse: that for some $l>N$ the opposite of (3.8) hold. We compute

$$
\sum_{i=0}^{l-1}\left[v\left(z_{i}^{l}, z_{i+1}^{l}\right)-\mu\right]=\sum_{i=0}^{N-1}\left[v\left(z_{i}^{l}, z_{i+1}^{l}\right)-\mu\right]+\sum_{i=N}^{l-1}\left[v\left(z_{i}^{l}, z_{i+1}^{l}\right)-\mu\right] .
$$

As discussed in (3.6), the second term exceeds $-(a-b)$, while by assumption the first one exceeds $\gamma+a-b$ so we get $\sum_{i=0}^{l-1}\left[v\left(z_{i}^{l}, z_{i+1}^{l}\right)-\mu\right] \geqslant \gamma+a-b-(a-b)=\gamma$ which is a contradiction.

Now let $l_{k} \rightarrow \infty$ be an increasing sequence of integers such that $z_{i}^{l_{k}} \underset{k \rightarrow \infty}{\rightarrow} z_{i}$ for every $i \geqslant 0$. Then we have

$$
m_{N}(\mathbf{z})=\sum_{i=0}^{N-1}\left[v\left(z_{i}, z_{i+1}\right)-\mu\right] \leqslant \liminf _{k \rightarrow \infty} \sum_{i=0}^{N-1}\left[v\left(z_{i}^{l_{k}}, z_{i+1}^{l_{k}}\right)-\mu\right] \leqslant \gamma+a-b
$$

and this holds for every $N \geqslant 1$. Thus conditions (1) and (2) are satisfied with $M=|\gamma|+a-b$.

\section{Comments and Examples}

In this section we comment on five subjects:

$a$. The question of computing $\mu$.

b. Some optimality considerations which are implied by Theorem 3.1 .

c. The compactness of $K$ : We display an example where $K$ is not compact and the conclusion of Theorem 3.1 is false.

$d$. The rectangular structure $K \times K$ : We display an example where admissible programs are such that $\left(z_{i}, z_{i+1}\right) \in \mathscr{D}$, where $\mathscr{D}$ is a compact subset of $R^{n} \times R^{n}$, not of a rectangular shape, and show that the conclusion of Theorem 3.1 is not valid there.

e. A relaxation iof the controllability assumption in some convex problems.

4a. The question of computing $\mu$ may be rather involved in general. In a special case when the cost of steering $x$ to $y$ equals that of steering $y$ to $x$ the computation is simple: 
Proposition 4.1. If $v(x, y)=v(y, x)$ for all $(x, y) \in K \times K$ then

$$
\mu=\min \{v(x, y):(x, y) \in K \times K\} .
$$

Proof. Denote $b=\min v(x, y)$. Then for every program $\mathrm{z}$ inequality $\frac{1}{N} \sum_{i=0}^{N-1} v\left(z_{i}, z_{i+1}\right) \geqslant b$ holds, and therefore $\lambda(N) \geqslant b$ for all $N \geqslant 1$. By the property $v(x, y)=v(y, x)$ we get that $\lambda(2)=b$, so that $\inf _{N \geqslant 1} \lambda(N)=\lambda(2)=b$ and $\mu=b$.

4b. As noted in the introduction, the reduction to finite costs provides us with easy means to compare programs. We shall define three concepts of optimality and explore the existence of optimal solutions.

Definition 4.2. We define the following partial order among programs: $x \leqslant y$ if $x_{0}=y_{0}$, and given an $\varepsilon>0$ there is an $N_{0}$ such that for all $N \geqslant N_{0}$

$$
\sum_{i=0}^{N} v\left(x_{i}, x_{i+1}\right)<\sum_{i=0}^{N} v\left(y_{i}, y_{i+1}\right)+\varepsilon .
$$

An overtaking optimal program is a program $\mathbf{s}$ such that $\mathbf{s} \leqslant \mathbf{z}$ for all $\mathbf{z}$ with $s_{0}=z_{0}$ (following Gale [3] and von Weizsacker).

Definition 4.3. We shall call a program $s$ a weakly optimal program if for every z satisfying $z_{0}=s_{0}$ and for every $\varepsilon>0$ there is a sequence of integers $N_{k} \rightarrow \infty$ such that

$$
\sum_{i=0}^{N_{k}} v\left(s_{i}, s_{i+1}\right)<\sum_{i=0}^{N_{k}} v\left(z_{i}, z_{i+1}\right)+\varepsilon \text { for all } N_{k} .
$$

(Also in this definition we follow the above mentioned authors.) As we shall see in example 4.7 weakly optimal programs may fail to exist.

Let us introduce the following concept

Definition 4.4. A program $s$ is called $\delta$-weakly optimal program if there exists a sequence of integers $N_{k} \rightarrow \infty$ such that for every $\mathbf{z}$ with $z_{0}=s_{0}$ the inequality $\sum_{i=0}^{N_{k}} v\left(s_{i}, s_{i+1}\right)<\sum_{i=0}^{N_{k}} v\left(z_{i}, z_{i+1}\right)+\delta$ holds for all large enough $k$.

Remark. The distinction between weakly optimal programs and $\delta$-weakly optimal programs is the following: If $\mathbf{s}$ is a weakly optimal program then it costs less, up to every $\varepsilon$, than any other program on an infinite number of intervals $\left[0, N_{k}\right]$. These intervals depend on the compared program. If $s$ is a $\delta$-weakly optimal program then all costs are compared up to $\delta$, and the sequence of intervals is 
independent of the program which is compared to $\mathbf{s}$. Therefore, a weakly optimal program is not necessarily a $\delta$-weakly optimal program.

Here is an immediate consequence of the reduction to finite costs:

Proposition 4.5. Given initial value $z_{0}=\alpha$ and $\delta>0$, there is a $\delta$-weakly optimal program $\mathbf{s}$ satisfying $s_{0}=\alpha$.

Proof. Define the functional $\Phi$ by

$$
\Phi(\mathbf{z})=\liminf _{N \rightarrow \infty} m_{N}(\mathbf{z}) .
$$

By Theorem 3.1, $\Phi(\mathbf{z})$ is bounded from below by $-M$. Therefore we can choose $\mathbf{z}_{\delta}$ with $\left(z_{\delta}\right)_{0}=\alpha$ such that $\Phi\left(\mathbf{z}_{\delta}\right)<\Phi(\mathbf{z})+\delta$ whenever $z_{0}=\alpha$. Let $N_{k} \rightarrow \infty$ be such that $m_{N_{k}}\left(\mathbf{z}_{\delta}\right) \underset{k \rightarrow \infty}{\rightarrow} \Phi\left(\mathbf{z}_{\delta}\right)$. Then $N_{k}$ is the sequence needed in Def. 4.4 and $\mathbf{z}_{\delta}$ is a $\delta$-weakly optimal program.

The following example exhibits the possibility of existence of a weakly optimal program along with nonexistence of an optimal program.

Example 4.6. Consider the function $v:[-1,1] \times[-1,1] \rightarrow R^{1}$, given by $v(x, y)=$ $(x+y)^{2}+x-y$. Since for every periodic program the cost is nonnegative, we deduce that $\mu \geqslant 0$. But computing the cost for the $\underset{N-1}{\operatorname{program}} z_{i}=0, i \geqslant 0$, leads to $\mu \leqslant 0$, so $\mu=0$. For a program $\mathbf{z}$ we have $m_{N}(\mathbf{z})=\sum_{i=0}\left(z_{i}+z_{i+1}\right)^{2}+z_{0}-z_{N}$. The minimization of $\sum_{i=0}^{N-1}\left(z_{i}-z_{i+1}\right)^{2}$ under the constraints $z_{0}=A$ and $z_{N}=B$ is obtained by

$$
z_{k}=(-1)^{k}(A+k \Delta), \quad 0 \leqslant k \leqslant N
$$

where $\Delta=\frac{B-A}{N}$ if $N$ is even, and $\Delta=-\frac{A+B}{N}$ if $N$ is odd.

We claim: If $\mathbf{z}$ is weakly optimal then $\mathbf{z}=\left\{z_{0},-z_{0}, z_{0},-z_{0}, \ldots\right\}$. The reason is the following: Suppose $z$ is weakly optimal and there is an $N \geqslant 1$ such that $z_{N} \neq(-1)^{N} z_{0}$. Assume $N$ is even and let $\delta=\left|\left(z_{N}-z_{0}\right) / N\right|$. We choose $N_{1}$ even and so large that $2 / N_{1}<\delta$. As mentioned above the minimum of $\sum_{i=0}\left(x_{i}+x_{i+1}\right)^{2}$ subject to $x_{0}=z_{0}, x_{N_{1}}=z_{N_{1}}$ is obtained by

$$
x_{k}=(-1)^{k}\left[z_{0}+k\left(\frac{z_{N_{1}}-z_{0}}{N_{1}}\right)\right] \text {, }
$$

but $\left\{x_{0}, x_{1}, \ldots, x_{N_{1}}\right\} \neq\left\{z_{0}, z_{1}, \ldots, z_{N_{1}}\right\}$ since

$$
\left|x_{N}-z_{0}\right|=N\left|\frac{z_{N_{1}}-z_{0}}{N_{1}}\right| \leqslant N \frac{2}{N_{1}}<N \delta=\left|z_{N}-z_{0}\right| \text {. }
$$


So we can replace the segment $\left\{z_{0}, \ldots, z_{N_{1}}\right\}$ by $\left\{x_{0}, \ldots, x_{N_{1}}\right\}$ (recall that $x_{N_{1}}=z_{N_{1}}$ ) and thus strictly lower the cost for all large times. Therefore the claim is proved.

We claim now that for $z_{0}>0$ the program $\left\{z_{0},-z_{0}, z_{0},-z_{0}, \ldots\right\}$ is weakly optimal. Otherwise, by (4.1), there is a program $\mathbf{s}$, and $\varepsilon>0$ and $N_{0}$ such that

$$
m_{2 N}(\mathbf{s}) \leqslant 2 z_{0}+\varepsilon
$$

and

$$
m_{2 N+1}(\mathbf{s}) \leqslant-\varepsilon
$$

both for all $N \geqslant N_{0}$. Substituting the explicit expression for $m_{N}(\mathbf{s})$ we get from (4.2) $\sum_{i=0}\left(s_{i}+s_{i+1}\right)^{2}+z_{0}-s_{2 N} \leqslant 2 z_{0}-\varepsilon$ and from this $s_{2 N} \geqslant \varepsilon-z_{0}$. Similarly, substitution of $m_{2 N+1}(\mathbf{s})$ in (4.3) leads to $s_{2 N+1} \geqslant \varepsilon+z_{0}$. Adding the last two inequalities we get $s_{2 N}+s_{2 N+1} \geqslant 2 \varepsilon$ which contradicts the finiteness of $\sum_{i=0}^{\infty}\left(s_{i}+\right.$ $\left.s_{i+1}\right)^{2}$. Thus $\mathbf{z}=\left\{z_{0},-z_{0}, z_{0},-z_{0}, \ldots,\right\}$ is weakly optimal.

Had there been an overtaking optimal program, it should have been this same program $\mathbf{z}$. But for the program $\mathbf{y}=\left\{z_{0},-\frac{1}{2} z_{0}, 0,0,0, \ldots\right\}$ we do not have $\mathbf{z} \leqslant \mathbf{y}$, since $m_{2 N}(\mathbf{z})=2 z_{0}>\frac{1}{2} z_{0}^{2}+z_{0}=m_{2 N}(\mathbf{y})$ for $N \geqslant 1$.

The next example will demonstrate nonexistence of weakly-optimal programs.

Example 4.7. Let $v:[-1,1] \times[-1,1] \rightarrow R^{1}, \quad v(x, y)=(x+y)^{2}+2\left(y^{2}-x^{2}\right)$. Reasoning as in Example 4.6, the only candidate to be a weakly optimal program is $\mathbf{z}=\left\{z_{0},-z_{0}, z_{0},-z_{0}, \ldots\right\}$. If we compare it with $\mathbf{y}=\left\{z_{0}, 0,0,0, \ldots\right\}$ we see that $m_{N}(\mathbf{y})=-z_{0}^{2}$ for every $N$, while $m_{N}(\mathbf{z})=0$, so $\mathbf{z}$ is not a weakly optimal program.

4c. We display now an example which shows that the compactness assumption concerning $K$ in Theorem 3.1 cannot be dispensed with.

Example 4.8. Let $v:[0, \infty) \times[0, \infty) \rightarrow R^{1}$ be the convex function given by $v(x, y)$ $=(y-x-1)^{2}+\frac{1}{x+1}$. We establish the following two facts:

(i) $\sum_{i=0}^{\infty} v\left(z_{i}, z_{i+1}\right)=\infty$ for every program $\left\{z_{i}\right\}_{i=0}^{\infty}$.

(ii) There is a program $\left\{z_{i}\right\}$ such that $\frac{1}{N} \sum_{i=0}^{N} v\left(z_{i}, z_{i+1}\right) \rightarrow 0$, namely: $\sum_{i=0}^{N} v\left(z_{i}, z_{i+1}\right)$ tends to infinity slower than any linear rate.

From these two facts, together with the simple consequence mentioned after Theorem 3.1 it follows that the conclusion of Theorem 3.1 is false in this example. 
We take any sequence $\left\{z_{i}\right)_{i=0}^{\infty}$ with $z_{i} \geqslant 0$, and suppose that $\sum_{i=0}^{\infty} v\left(z_{i}, z_{i+1}\right)$ $<\infty$. Then clearly $z_{i+1}-z_{i}-1 \rightarrow 0$, and therefore $z_{i+1}<z_{\dot{\alpha}}+2$ for $i>i_{0}$. From this follows that $z_{i}<z_{i_{0}}+2\left(i-i_{0}\right)$ for all $i>i_{0}$, so that $\sum_{i=0}^{\infty} \frac{1}{z_{i}+1}=\infty$, consequently $\sum_{i=0}^{\infty} v\left(z_{i}, z_{i+1}\right)=\infty$, contradicting the assumption. So (i) is established.

Considering the sequence $z_{i}=i, i=0,1,2, \ldots$ we get $\sum_{i=0}^{N-1} v\left(z_{i}, i_{i+1}\right)=\sum_{i=0}^{N} \frac{1}{i}$, and (ii) is satisfied for this sequence.

4d. Instead of considering functions $v: K \times K \rightarrow R^{1}$ for compact sets $K \subset R^{n}$, we could have considered functions $v: \mathscr{D} \rightarrow R^{1}$, where $\mathscr{D}$ is compact in $R^{n} \times R^{n}$. Then a program is defined as a sequence $\left\{z_{i}\right\}_{i=0}^{\infty}$ satisfying $\left(z_{i}, z_{i+1}\right) \in \mathscr{D}$ for all $i \geqslant 0$. The following example demonstrates the fact that in general the conclusion of Theorem 3.1 is false unless the set $\mathscr{D}$ has a rectangular shape $K \times K$.

The way by which Example 4.9 does not satisfy the conclusion of Theorem 3.1 is this: We show that for $\mu \geqslant 1$ all the modified cost flows diverge to $-\infty$, while for $\mu<1$ all of them diverge to $+\infty$. Thus it is impossible to find a $\mu$ satisfying the conclusion of Theorem 3.1

Example 4.9. Let $\mathscr{D} \subset R^{2}$ be the following line

$$
\mathscr{D}=\left\{(x, y): y=\frac{1}{2}(x+1), \frac{1}{2} \leqslant x \leqslant 1\right\}
$$

and let $v: \mathscr{D} \rightarrow R^{1}$ be given by

$$
v(x, y(x))= \begin{cases}{\left[\log _{2}(1-x)\right]^{-1}+1} & \text { if } \frac{1}{2} \leqslant x<1 \\ 1 & \text { if } x=1\end{cases}
$$

Then it is easy to see that $v$ is a continuous and convex function on $\mathscr{D}$.

We take any initial value $\frac{1}{2} \leqslant z_{0}<1$. If we choose $\mu<1$, then the modified cost flow will diverge to $+\infty$ : to see this we note that the only admissible program starting at $z_{0}$ satisfies $z_{k} \rightarrow 1$, therefore $\left[v\left(z_{k}, z_{k+1}\right)-\mu\right] \rightarrow 1-\mu>0$, so we get $\sum_{k=0}^{\infty}\left[v\left(z_{k}, z_{k+1}\right)-\mu\right]=\infty$.

On the other hand, if we choose $\mu \geqslant 1$, then $\sum_{k=0}^{\infty}\left[v\left(z_{k}, z_{k+1}\right)-\mu\right]$ $\leqslant \sum_{k=0}^{\infty}\left[\log _{2}\left(1-z_{k}\right)\right]^{-1}$. The latter series diverges to $-\infty$. To see this we note that if $x$ satisfies $1-1 / 2^{p} \leqslant x<1-1 / 2^{p+1}$ for some integer $p$, then for $y$ such that $(x, y) \in \mathscr{D}$ we have $y=\frac{1}{2}(x+1)$ and therefore

$$
1-\frac{1}{2^{p+1}} \leqslant y<1-\frac{1}{2^{p+2}} \text {. }
$$


If $1-1 / 2^{p} \leqslant z_{0}<1-1 / 2^{p+1}$ then $\left[\log _{2}\left(1-z_{0}\right)\right]^{-1}<-(p+1)^{-1}$ and by the above calculation we get $\left[\log _{2}\left(1-z_{k}\right)^{-1}<-(p+k+1)^{-1}\right.$ and the assertion is proved.

To conclude: the cost flows diverge to infinity slower than linear function $L(N)=N$ but faster than any linear function $L(N)=\lambda N$ with $\lambda<1$.

The same phenomenon with the same proof occurs in the following example: $\mathscr{D}$ is the triangle $\left\{(x, y) \in R^{2}: x \leqslant y \leqslant \frac{1}{2}(x+1), \frac{1}{2} \leqslant x \leqslant 1\right\}$

$$
v(x, y)=\left\{\begin{array}{lll}
{\left[\log _{2}(y-x)\right]^{-1}+1} & y>x, & (x, y) \in \mathscr{D} \\
1 & y=x, & (x, y) \in \mathscr{D} .
\end{array}\right.
$$

4e. We demonstrate now that the pathology described in Section $4 \mathrm{~d}$ cannot occur in a certain special case. In Example 4.9, the minimum of $v(z, z)$ was achieved in a boundary point of $\mathscr{D}$, and this we want to prevent now.

We prove the following: Let $\mathscr{D}$ be a compact and convex subset of $R^{n} \times R^{n}$, $v: \mathscr{D} \rightarrow R^{1}$ is a convex function, $(d, d) \in$ int $\mathscr{D}, v(z, z) \geqslant v(d, d)$ for all $(z, z) \in \mathscr{D}$ and the initial value $z_{0}$ can be steered to $d$ in a finite time i.e. there exists a program $z_{0}=x_{0}, x_{1}, \ldots, x_{N}$ with $x_{N}=d$ and $\left.\left(x_{i}, x_{i+1}\right) \in \mathscr{D}\right)$. Under these conditions the conclusion of Theorem 3.1 is valid.

We outline the proof: Let $U \subset \mathscr{D}$ be a ball around $(d, d)$. Recall that for a real valued function $\varphi: \mathscr{B} \rightarrow R^{1}$ the set epi $\varphi$ is defined by $\{(r, x): x \in \mathscr{B}$, $r \geqslant \varphi(x)\}$. Let $A \subset R^{n} \times R^{n} \times R^{1}$ be defined by $A=($ epi $v) \cup$ $\{(x, x, v(d, d)):(x, x) \in U\}$ and let $w(x, y)$ be the function whose epigraph is $\operatorname{conv} A$ (namely the closed convex hull of $A$ ). Then it is easy to see that $w(z, z) \geqslant v(d, d)$ for all $(z, z) \in \mathscr{D}$.

Evidently as epi $v \subseteq$ epi $w$, the inequality $w(x, y) \leqslant v(x, y)$ holds for every $(x, y) \in \mathscr{D}$. Since $(d, d)$ is an interior point of $\mathscr{D}$, and $w: \mathscr{D} \rightarrow R^{1}$ is convex, there is a subgradient vector for $w$ at $(d, d)$. From the fact that $w(z, z)=v(d, d)$ for every $(z, z) \in U$ it follows that the subgradient vector at $(d, d)$ is of the form $(\eta,-\eta)$, where $\eta \in R^{n}$. Thus we get the inequality

$$
v(x, y) \geqslant w(x, y) \geqslant v(d, d)+\left\langle\eta^{+}, x-y\right\rangle
$$

for all $(x, y) \in \mathscr{D}$. Here $\left\langle a^{+}, b\right\rangle$ denotes the scalar multiplication between two vectors $a$ and $b$ in $R^{n}$. For every program $\mathbf{z}$ we have

$$
\sum_{i=0}^{N-1}\left[v\left(z_{i}, z_{i+1}\right)-v(d, d)\right] \geqslant\left\langle\eta^{+}, z_{0}-z_{N}\right\rangle
$$

and the right hand side has a uniform bound over all $\mathbf{z}$ and all $N$. On the othe hand, by assumption, there is a program $\mathrm{z}$ such that $z_{N_{0}}=d$ for some $N_{0}$. If we choose $z_{N}=d$ for all $N>N_{0}$ then the modified flow $\sum_{i=0}^{N^{0}}\left[v\left(z_{i}, z_{i+1}\right)-v(d, d)\right]$ is bounded.

We note therefore that the conclusion of Theorem 3.1 holds and furthermore the constant $\mu$ is equal to the value $v(d, d)$. 


\section{A Useful Representation}

In this section we shall confine ourselves to continuous cost functions $v(x, y)$. (We shall formulate, but not prove, the analogous result for $v(x, y)$ lower semicontinuous.)

We intend to represent every continuous function on a compact rectangular domain in a special way, which has a close relation to the reduction to finite costs.

Proposition 5.1. Let $K \subset R^{n}$ be compact and $v: K \times K \rightarrow R^{1}$ be a continuous function. Then $v$ can be represented as follows:

$$
v(x, y)=\mu+\pi(x)-\pi(y)+\theta(x, y) \text { where }
$$

(a) $\mu$ is a constant;

(b) $\pi: K \rightarrow R^{1}$ and $\theta: K \times K \rightarrow R^{1}$ are continuous functions;

(c) $\theta$ is nonnegative and $E(x)=\{y \in K: \theta(x, y)=0\}$ is nonempty for every $x \in K$.

Comment. Knowing that $v$ has this representation enables us to give a simple proof to Theorem 3.1. In fact we take $M=2 \max _{z \in K}|\pi(z)|$ and then for every program z:

$$
\sum_{i=0}^{N-1}\left[v\left(z_{i}, z_{i+1}\right)-\mu\right]=\sum_{i=0}^{N-1} \theta\left(z_{i}, z_{i+1}\right)+\pi\left(z_{0}\right)-\pi\left(z_{N}\right) \geqslant-M,
$$

while for a program chosen by $z_{k+1}^{*} \in E\left(z_{k}^{*}\right)$

$$
\left|\sum_{i=0}^{N-1}\left[v\left(z_{i}^{*}, z_{i+1}^{*}\right)-\mu\right]\right|=\left|\pi\left(z_{N}^{*}\right)-\pi\left(z_{0}^{*}\right)\right| \leqslant M .
$$

Our method, however, is the other way around: we use the existence of the reduction to finite costs in Theorem 3.1 to prove Proposition 5.1.

Proof of Proposition 5.1. Let $\mu$ be as guaranteed in Theorem 3.1 and defined in (3.2). Define $\pi: K \rightarrow R^{1}$ by

$$
\pi(x)=\inf _{\substack{\mathbf{z} \\ z_{0}=x}}\left\{\liminf m_{N}(\mathbf{z})\right\}
$$

Given any pair $(x, y) \in K \times K$ we claim that $\pi(x) \leqslant[v(x, y)-\mu]+\pi(y)$. The reason is: If we confine ourselves to programs $\mathbf{z}$ such that $z_{0}=x, z_{1}=y$ and compute the right hand side of (5.2) over these programs only, then we get $[v(x, y)-\mu]+\pi(y)$. Oi course $\pi(x)$ is not greater than this vaiue. 
We define $\theta: K \times K \rightarrow R^{1}$ by

$$
\theta(x, y)=v(x, y)-\mu+\pi(y)-\pi(x)
$$

and get (5.1) with $\theta$ nonnegative. The uniform continuity of $v$ on $K \times K$ implies the continuity of $\pi$, and thus, by (5.3), the function $\theta$ is continuous too.

It only remains to prove that $E(x)$ is nonempty for every $x \in K$. Suppose to the contrary that for some $x \in K$

$$
\min \{\theta(x, y): y \in K\}=\delta>0 .
$$

There is a program $\mathbf{z}$ such that: $z_{0}=x$ and $\liminf _{N \rightarrow \infty} m_{N}(\mathbf{z})<\pi(x)+\frac{1}{2} \delta$. We compute:

$$
\begin{aligned}
\pi(x)+\frac{1}{2} \delta> & \liminf _{N \rightarrow \infty} m_{N}(\mathbf{z})=\left[\theta\left(x, z_{1}\right)+\pi(x)-\pi\left(z_{1}\right)\right] \\
& +\liminf _{N \rightarrow \infty} \sum_{i=1}^{N}\left[v\left(z_{i}, z_{i+1}\right)-\mu\right] \\
\geqslant & {\left[\delta+\pi(x)-\pi\left(z_{1}\right)\right]+\pi\left(z_{1}\right) }
\end{aligned}
$$

So $\pi(x)+\frac{1}{2} \delta>\pi(x)+\delta$, a contradiction; hence (5.4) is false.

For $v(x, y)$ which are merely lower semicontinuous, we have the following analogue of Proposition 5.1:

Proposition 5.1'. Let $v: K \times K \rightarrow R^{1}$ be bounded and lower semicontinuous. Let $\left(c^{\prime}\right)$ be the condition:

( $\left.c^{\prime}\right) \theta: K \times K \rightarrow R^{1}$ is nonnegative and for every $X \in K, \inf _{y \in K}\{\theta(x, y): y \in$ $K\}=0$. Then $v$ admits the representation (5.1) with $\left(c^{\prime}\right)$ replacing $(c)$.

The proof is essentially the same as that for Proposition (5.1) with only minor modifications. We leave out the details.

Following Gale [3], let us introduce the following terminology:

Definition 5.2. A program $\mathbf{z}$ is called a good program if $\left\{m_{N}(\mathbf{z})\right\}_{N=1}^{\infty}$ forms a bounded sequence.

We apply propositions 5.1 and $5.1^{\prime}$ to show how one can build good programs. Generally, for lower semicontinuous functions, this can be achieved by choosing $\mathrm{z}$ so that $\theta\left(z_{i}, z_{i+1}\right)<1 / 2^{i}$. If $v(x, y)$ is continuous this can be done in a dynamical programming type way, by constructing a $\mathbf{Z}$ which satisfies $z_{i+1} \in$ $E\left(z_{i}\right)$. For a program so generated we get

$$
\left|\sum_{i=0}^{N-1}\left[v\left(z_{i}, z_{i+1}\right)-\mu\right]\right|=\left|\pi\left(z_{0}\right)-\pi\left(z_{N}\right)\right| \leqslant M,
$$

proving that $\mathbf{z}$ is a good program. 
The representation (5.1) always holds for continuous $v(x, y)$. If it happens that the cost function $v(x, y)$ has the following form, which is more restrictive than that in (5.1), then we can establish the existence of overtaking optimal programs (recall definition 4.2).

Proposition 5.3. Let $v: K \times K \rightarrow R^{1}$ be a continuous function which admits $a$ representation $v(x, y)=\mu+\pi(x)-\pi(y)+\theta(x, y)$, where

(i) $\mu$ is a constant, $\pi: K \rightarrow R^{1}$ and $\theta: K \times K \rightarrow R^{1}$ are continuous functions.

(ii) $\theta$ is nonnegative and there is an element $p \in K$ such that $(x, y)=(p, p)$ if and only if $\theta(x, y)=0$.

Then for every initial value there is an overtaking optimal program.

Proof. If $\mathbf{z}$ is a good program then there is a bound on

$$
\left\{\pi\left(z_{0}\right)-\pi\left(z_{N}\right)+\sum_{i=0}^{N-1} \theta\left(z_{i}, z_{i+1}\right)\right\}_{N=1}^{\infty},
$$

consequently $\theta\left(z_{i}, z_{i+1}\right) \rightarrow 0$, and condition (ii) implies that $z_{i} \rightarrow p$. So for every good program $z$ we have

$$
\sum_{i=0}^{\infty}\left[v\left(z_{i}, z_{i+1}\right)-\mu\right]=\pi\left(z_{0}\right)-\pi(p)+\sum_{i=0}^{\infty} \theta\left(z_{i}, z_{i+1}\right) .
$$

The functional $\mathbf{z} \rightarrow \sum_{i=0}^{\infty} \theta\left(z_{i}, z_{i+1}\right)$ is lower semicontinuous as a functional on the set of programs endowed with the topology of pointwise convergence (if $\mathbf{z}^{k} \rightarrow \mathbf{z}$ and $\sum_{i=0}^{\infty} \theta\left(z_{i}, z_{i+1}\right)=\alpha$ then given $\varepsilon>0, \sum_{i=0}^{N} \theta\left(z_{i}, z_{i+1}\right)>\alpha-\varepsilon$ for some $N$, so $\sum_{i=0}^{N} \theta\left(z_{i}^{k}, z_{i+1}^{k}\right)>\alpha-2 \varepsilon$ for $k$ large). Let $\mathbf{s}$ be a program which minimizes this functional. Clearly $\mathbf{s}$ is a good program so it minimizes the functional $\mathbf{z}$ $\rightarrow \sum_{i=0}^{\infty}\left[v\left(z_{i}, z_{i+1}\right)-\mu\right]$ too and the overtaking optimality of $\mathbf{s}$ follows.

As a corollary we get the following result, concerning convex functions $v(x, y)$ :

Corollary 5.4. Let $v(x, y)$ be strictly convex in $(x, y)$, such that the function $z \rightarrow v(z, z)$ has a minimum at $p \in K$. Then for every initial value there is a unique overtaking optimal program.

Proof. By (4.4) there is a $\eta \in R^{n}$ such that $v(x, y) \geqslant v(p, p)+\left\langle\eta^{+}, x-y\right\rangle$. Define $\mu=v(p, p)$ and $\theta(x, y)=v(x, y)-\mu-\left\langle\eta^{+}, x-y\right\rangle$. Then $\theta$ is nonnegative and strictly convex. Since $\theta(p, p)=0$ it follows that whenever $(x, y) \neq(p, p)$ then $\theta(x, y)>0$.

Example 5.5. Let $v(x, y)=\left(1+x^{2}+y^{2}\right)^{1 / 2}-x^{2}, 0 \leqslant x \leqslant 1$ and $0 \leqslant y \leqslant 1$. We claim that there is an overtaking optimal program for every initial value. Denote 
$r^{2}=x^{2}+y^{2}$ and let $\rho=\left(1+r^{2}\right)^{1 / 2}$. Then we can write $v(x, y)=\frac{1}{2}[1+\rho(2-\rho)$ $\left.+y^{2}-x^{2}\right]$ which may be given the form required in Proposition 5.2, by $\mu=\sqrt{3}$ $-1, \pi(x)=-\frac{1}{2} x$ and $\theta(r)=\frac{1}{2}[\rho(2-\rho)+3-2 \sqrt{3}]$. We have $\theta(r)>0$ for $1 \leqslant r$ $<\sqrt{2}$ and $\theta(\sqrt{2})=0$, so the claim follows from Proposition 5.3.

\section{An Application: Costs with Discounting Factors}

It is customary in mathematical economics to introduce into the costs expressions a discounting factor. Usually this factor is chosen to be an exponentially decreasing function, which reflects the result of a constant interest rate. The introduction of an exponentially decreasing discounting factor induces on the interval $[0, \infty)$ a finite measure, and in many cases makes the set of trajectories compact in a suitable topology.

We discuss here a discounting factor which tends to zero, but may do so very slowly. Therefore compactness arguments which work for the exponential decay would not work. We consider the following problem: $v: K \times K \rightarrow R^{1}$ is a continuous function. $\left\{\alpha_{i}\right\}_{i=0}^{\infty}$ is a sequence of positive numbers converging monotonically to zero. For a program $\mathbf{z}$ we study the cost flow $\left\{\sum_{i=0}^{N} \alpha_{i} v\left(z_{i}, z_{i+1}\right\}_{N=0}^{\infty}\right.$.

Anologous to Definition 4.2 we define here an overtaking optimal program as a program $\left\{s_{K}\right\}_{k=0}^{\infty}$ such that for every $\varepsilon>0$ and every other program $\mathbf{z}$ with $z_{0}=s_{0}$ we have

$$
\sum_{k=0}^{N} \alpha_{k} v\left(s_{k}, s_{k+1}\right) \leqslant \sum_{k=0}^{N} \alpha_{k} v\left(z_{k}, z_{k+1}\right)+\varepsilon
$$

for all $N$ large enough.

Using the representation formula (5.1) we can establish the existence of an overtaking optimal program for the present problem. We emphasize that $\left\{\alpha_{i}\right\}$ may decrease to zero very slowly so that still $\sum_{i=0}^{\infty} \alpha_{i} v\left(z_{i}, z_{i+1}\right)=\infty$ for every program.

Theorem 6.1. Let $v: K \times K \rightarrow R^{1}$ be continuous. Let $\left\{\alpha_{i}\right\}_{i=0}^{\infty}$ be a nonincreasing sequence with $\alpha_{i} \rightarrow 0$ as $i \rightarrow \infty$. Then for every initial value $z_{0}$ there is an overtaking optimal program $\mathbf{s}$ with $s_{0}=z_{0}$.

Proof. Using (5.1) we compute for $\mathbf{z}$

$$
\begin{aligned}
\sum_{i=0}^{N-1} \alpha_{i}\left[v\left(z_{i}, z_{i+1}\right)-\mu\right]= & \sum_{i=0}^{N-1} \alpha_{i} \theta\left(z_{i}, z_{i+1}\right)+\alpha_{0} \pi\left(z_{0}\right)-\alpha_{N-1} \pi\left(z_{N}\right) \\
& +\sum_{i=1}^{N-1}\left(\alpha_{i}-\alpha_{i-1}\right) \pi\left(z_{i}\right) .
\end{aligned}
$$


The series $\sum_{i=0}^{\infty} \alpha_{i} \theta_{i}\left(z_{i}, z_{i+1}\right)$ is either convergent or diverges to infinity, by positivity, and the series $\sum_{i=1}^{\infty}\left(\alpha_{i}-\alpha_{i-1}\right) \pi\left(z_{i}\right)$ converges, since $\sum\left(\alpha_{i}-\alpha_{i-1}\right)$ converges absolutely. So we denote

$$
\begin{aligned}
& \varphi(z)=\sum_{i=0}^{\infty} \alpha_{i}\left[v\left(z_{i}, z_{i+1}\right)-\mu\right] \\
& \varphi_{1}(z)=\sum_{i=1}^{\infty}\left(\alpha_{i}-\alpha_{i-1}\right) \pi\left(z_{i}\right) \\
& \varphi_{2}(z)=\sum_{i=0}^{\infty} \alpha_{i} \theta\left(z_{i}, z_{i+1}\right)
\end{aligned}
$$

and have the relation $\varphi(\mathbf{z})=\varphi_{1}(\mathbf{z})+\varphi_{2}(\mathbf{z})+\alpha_{0} \pi\left(z_{0}\right)$. We consider the set of programs endowed with the topology of pointwise convergence. We claim that in this topology both $\varphi_{1}$ and $\varphi_{2}$ are lower semicontinuous.

Lower semicontinuity of $\varphi_{2}$ follows since $\theta(x, y)$ is positive and continuous. (Compare with the proof of proposition 5.2)

Lower semicontinuity of $\varphi_{1}$ : Let $\mathbf{z}^{k} \rightarrow \mathbf{z}$ and let $\varepsilon>0$. Then there is an $N$ such that for every program $\left|\sum_{i=N}^{\infty}\left(\alpha_{i}-\alpha_{i-1}\right) \pi\left(s_{i}\right)\right|<\varepsilon / 3$. For this $N$

$$
\begin{aligned}
\varphi_{1}(\mathbf{z})-\varphi_{1}\left(\mathbf{z}^{k}\right)= & \sum_{i=N}^{\infty}\left(\alpha_{i}-\alpha_{i-1}\right) \pi\left(z_{i}\right)-\sum_{i=N}^{\infty}\left(\alpha_{i}-\alpha_{i-1}\right) \pi\left(z_{i}^{k}\right) \\
& +\sum_{i=1}^{N-1}\left(\alpha_{i}-\alpha_{i-1}\right)\left[\pi\left(z_{i}\right)-\pi\left(z_{i}^{k}\right)\right]
\end{aligned}
$$

If $k$ is large enough the third term is less than $\varepsilon / 3$ so that $\varphi(\mathbf{z})<\varphi\left(\mathbf{z}^{k}\right)+\varepsilon$ for all large $k$.

Thus we conclude that $\varphi$ itself is lower semicontinuous in this topology and by compactness of $K$ and boundedness below of $\varphi$ there is a program $\mathbf{s}$ such that $\varphi(\mathbf{s}) \leqslant \varphi(\mathbf{z})$ for all $\mathbf{z}$. To prove (6.1), given any program $\mathbf{z}$ and an $\varepsilon>0$ there is $N_{\varepsilon}$ such that for all $N>N_{\varepsilon}$

$$
\sum_{i=0}^{N} \alpha\left[v\left(s_{i}, s_{i+1}\right)-\mu\right]<\sum_{i=0}^{N} \alpha_{i}\left[v\left(z_{i}, z_{i+1}\right)-\mu\right]+\varepsilon
$$

Now (6.1) follows by adding $\mu \sum_{i=0}^{N} \alpha_{i}$ to both sides of the inequality.

We shall employ now Theorem 6.1 to study continuous time control systems which are represented by an ordinary differential equation, and whose cost 
integrand contains a discounting factor. Namely, we consider a system

$$
\left\{\begin{array}{l}
\dot{z}=f(z, w) \\
c_{T}(u)=\int_{0}^{T} f_{0}(z(t), u(t)) \varphi(t) d t
\end{array}\right.
$$

where $z(t) \in K, u(t) \in \Omega$ for $0 \leqslant t \leqslant T$, while $K$ and $\Omega$ are compact subsets of $R^{n}$ and $R^{m}$ respectively, and $\varphi(t)$ is a positive scalar function which decreases monotonically to zero. The admissible controls are all the measurable functions $u(t)$ for which the constraints $u(t) \in \Omega$ and $z(t) \in K$ are satisfied (where $z$ and $u$ are related as in (6.5)). We assume the following:

(1) The functions of $f$ and $f_{0}$ are continuous on $K \times \Omega$.

(2) A controllability assumption: For every $(x, y) \in K \times K$ there is an admissible control $u(t)$ with a corresponding trajectory $z(t)$ which satisfies $z(0)=x, z(1)=y$.

(3) The function $v(x, y)$ defined on $K \times K$ by

$$
\begin{gathered}
v(x, y)=\inf \left\{\int_{0}^{1} f_{0}(z(t), u(t) d t: \dot{z}=f(z, u), z(t) \in K,\right. \\
u(t) \in \Omega, z(0)=x, z(1)=y\}
\end{gathered}
$$

is continuous on $K \times K$.

(4) The function

$$
\begin{gathered}
w_{T}(x, y)=\min \left\{\int_{T}^{T+1} f_{0}(z(t), u(t)) \varphi(t) d t: \dot{z}=f(z, u), z(t) \in K,\right. \\
u(t) \in \Omega, z(T)=x, z(T+1)=y\}
\end{gathered}
$$

is well defined (namely, the minimum is attained by a certain admissible control), and is continuous on $K \times K$. This for every $T>0$.

Remark. There can be given explicit assumptions concerning $f$ and $f_{0}$ which guarantee the validity of (3) and (4). However, they seem to be too restrictive and we prefer the implicit assumption (3) and (4).

Assumption (3) guarantees a constant $\mu$ with the properties which are described in Theorem 3.1.

Theorem 6.2. Let the control system (6.4) satisfy assumptions (1), (2), (3) and (4). Then for every initial value $z_{0} \in K$ there exists an overtaking optimal solution $z^{*}(t)$ satisfying $z^{*}(0)=z_{0}$.

To prove Theorem 6.2 we shall need the following Lemma: 
Lemma 6.3. Given an $\varepsilon>0$ then there is a time $T_{0}>0$ such that

$$
\int_{T_{1}}^{T_{2}}\left[f_{0}(z(t), u(t))-\mu\right] \varphi(t) d t>-\varepsilon \text { for every } T_{2}>T_{1} \geqslant T_{0}
$$

for every $z(t) \in K$ and every $u(t) \in \Omega$.

Proof. It is enough to prove the claim for $T_{1}, T_{2}$ integers. Denote by $\psi(t)$ the function $\psi(t)=\varphi(k)$ for $k \leqslant t<k+1$. For all integers $M>N$ and functions $z(t) \in K, u(t) \in \Omega$ the following equality holds:

$$
\begin{aligned}
& \int_{N}^{M} {\left[f_{0}(z, u)-\mu\right] \varphi(t) d t } \\
& \quad=\int_{N}^{M}\left[f_{0}(z, u)-\mu\right][\varphi(t)-\psi(t)] d t+\int_{N}^{M}\left[f_{0}(z, u)-\mu\right] \psi(t) d t .
\end{aligned}
$$

Let $A$ be a bound on $\left|f_{0}(z, u)-\mu\right|$, and notice that $\int_{N}^{M}|\varphi(t)-\psi(t)| d t \leqslant \varphi(N)$ so the first term in the last equation exceeds $[-A \varphi(N)]$. For the second we estimate

$$
\begin{aligned}
\int_{N}^{M}\left[f_{0}(z, u)-\mu\right] \psi(t) d t & \geqslant \sum_{k=N}^{M-1} \varphi(k) \int_{k}^{k+1}\left[f_{0}(z, u)-\mu\right] d t \\
& \geqslant \sum_{k=N}^{M-1} \varphi(k)[v(z(k), z(k+1))-\mu] .
\end{aligned}
$$

By (6.2) the last expression can be estimated by

$$
\varphi(N) \pi\left(z_{N}\right)-\varphi(M-1) \pi\left(z_{M}\right)+\sum_{i=N+1}^{M-1}[\varphi(i)-\varphi(i-1)] \pi(z(i))
$$

and by boundedness of $|\pi(z)|$ and $\varphi(t) \rightarrow 0$ this estimate is less than $\varepsilon$ in absolute value if $N$ is large enough, thus the claim is proved.

Proof of Theorem 6.2. From (6.6) it follows that for every $z(t) \in K, u(t) \in \Omega$, the expression $\int_{0}^{\infty}\left[f_{0}(z(t), u(t))-\mu\right] \varphi(t)$ either concerges or diverges to infinity. Moreover, for good programs this integral is finite (because the convergence of $\sum_{i=0}^{\infty} \theta\left(z_{i}, z_{i+1}\right)$ clearly implies that of $\sum_{i=0}^{\infty} \alpha_{i} \theta\left(z_{i}, z_{i+1}\right)$ if $\left.\alpha_{i} \rightarrow 0\right)$. Let $m=$ $\inf \int_{0}^{\infty}\left[f_{0}(z, u)-\mu\right] \varphi(t) d t$, where $u$ and $z$ are related by the differential equation $\dot{z}=f(z, u)$ and initial condition $z(0)=x \in K$, and where the infimum is taken over all admissible controls. Then there are admissible pairs $\left(z_{k}, u_{k}\right)$ such that

$$
\int_{0}^{\infty}\left[f_{0}\left(z_{k}(t), u_{k}(t)\right)-\mu\right] \varphi(t) d t=m_{k} \rightarrow m .
$$

It can be assumed that $z_{k}(i) \underset{k \rightarrow \infty}{\rightarrow} z(i)$ for all $i \geqslant 0$, and by assumption (4), define $z(t)$ in $[i, i+1]$ as a minimal solution of $\int_{i}^{i+1} f_{0}(x(t), u(t)) \varphi(t) d t$ satisfying 
$x(i)=z(i), \quad x(i+1)=z(i+1)$. Now by assumption (4) (the continuity of $\left.w_{N}(x, y)\right)$ and Lemma 6.3, it follows that, given $\varepsilon>0, \int_{0}^{T}\left[f_{0}(z(t), u(t))-\mu\right] \varphi(t)$ $\leqslant m+\varepsilon$ for all large $T$, so we conclude $\int_{0}^{\infty}\left[f_{0}(z(t), u(t))-\mu\right] \varphi(t) d t=m$ and $z(t)$ is an overtaking optimal solution.

Remark. This result can be viewed a little differently: Given an initial value $x \in K$ there exists a constant $m$ so that for every admissible pair $(z(t), u(t))$ with $z(0)=x$ the following limit exists and satisfies

$$
\lim _{T \rightarrow \infty}\left\{\int_{0}^{T} f(z(t), u(t)) \varphi(t) d t-\left[m+\mu \int_{0}^{T} \varphi(t) d t\right]\right\} \geqslant 0
$$

while there exists an admissible pair $\left(z^{*}(t)^{*}, u(t)\right)$ with $z^{*}(0)=x$ for which the equality holds. The function $T \rightarrow m+\mu \int_{0}^{T} \varphi(t) d t$ expresses the minimal cost growth as $T \rightarrow \infty$.

\section{An Application}

Bellman and Bucy considered in [1] the minimization of

$$
c_{T}[u]=\frac{1}{2} \int_{0}^{T}\left[u^{2}(t)+L(z(t))\right] d t
$$

where $t \rightarrow u(t)$ and $t \rightarrow z(t)$ are scalar functions, $u$ is measurable on $[0, \infty)$, and $u, z$ are constrained by $\dot{z}=f(z)+u$ and $z(0)=x$, for a certain $x \in R^{1}$. They studied this problem from several aspects and raised two questions which we quote as follows:

(1) When does the problem for $T=\infty$ make sense?

(2) When it does, are the optimal states and controls for infinite $T$ the limits of those for finite $T$ ?

The analysis in [1] is carried under quite restrictive assumptions. We shall treat this problem under more general assumptions, which are the following:

(i) The function $f$ is continuous and there exists a constant $a>0$ such that $|f(x)| \leqslant a(|x|+1)$ for all $-\infty<x<\infty$.

(ii) $L(x) \rightarrow \infty$ as $|x| \rightarrow \infty$ and $[f(x)]^{2}+L(x)$ is strictly convex. Conditions (i) and (ii) are fulfilled under the assumptions made in [1].

We define

$$
v_{0}(x, y)=\inf \left\{\int_{0}^{1}\left[u^{2}(t)+L(z(t))\right] d t: \dot{z}=f(z)+u, z(0)=x, z(1)=y\right\} .
$$

Substituting $u=\dot{z}-f(z)$ in the cost integral, we get

$$
v_{0}(x, y)=\inf \left\{\int_{0}^{1}\left[(\dot{z}-f(z))^{2}+L(z)\right] d t: z(0)=x, z(1)=y\right\} .
$$


Taking $F(z)$ such that $\frac{d F}{d z}=f(z)$, defining $M(z)=[f(z)]^{2}+L(z)$ and

$$
v(x, y)=\min \left\{\int_{0}^{1}\left[\dot{z}^{2}+M(z)\right] d t: z(0)=x, z(1)=y\right\}
$$

we get $v_{0}(x, y)=v(x, y)+2 F(x)-2 F(y)$.

By (ii) $M(z)$ is strictly convex, therefore $v(x, y)$ is strictly convex. Therefore it is continuous on $R^{1} \times R^{1}$, implying the continuity of $v_{0}(x, y)$ on $R^{n} \times R^{n}$.

The system in this problem is controllable since for every $(x, y) \in R^{1} \times R^{1}$ we can choose $z(t)=x+t(y-x), u(t)=y-x-f(x+t(y-x))$.

We claim that $v_{0}(x, y) \rightarrow \infty$ as $|x|+|y| \rightarrow \infty$. Let $M>0$, let $u(t)$ be such that

$$
\int_{0}^{1} u^{2}(t) d t \leqslant M
$$

and let $\dot{z}=f(z(t))+u(t), 0 \leqslant t \leqslant 1$. Take $z(0)=x$ to be a large positive number, then $\frac{d z}{d t} \geqslant-a z+u-a$, which yields $z(t) \geqslant e^{-a t} x+\int_{0}^{t} e^{-a(t-\tau)} u(\tau) \mathrm{d} \tau-1$ so we get $z(t) \geqslant e^{-a t} x-M^{1 / 2}-1$, by (7.1) and the Cauchy-Schwarz inequality. So if $x$ is large enough, we have (by $L(z) \rightarrow \infty$ as $z \rightarrow \infty$ ) that $L(z(t))>M$ for all $0 \leqslant t \leqslant 1$. From this, together with (7.1), it follows that $v_{0}(x, y) \geqslant M$ for all large enough $x$ and all $y$. Similar computation for large positive $y$, and for large negative $x$ or $y$ conclude the proof of the claim.

By the assertion that will be stated and proved in section 8 , it follows that a compact $K \subset R^{1}$ exists such that only trajectories $z(t) \in K$ need to be considered in the optimization problem.

Now returning to the equality $v_{0}(x, y)=v(x, y)+2 F(x)-2 F(y)$, and recalling that $v(x, y)$ is strictly convex, it follows from comment $4 e$ and Corollary 5.4 that $v(x, y)$, hence $v_{0}(x, y)$ is represented as required in Proposition 5.3, consequently there exists an optimal overtaking solution to the problem. Because of strict convexity it is unique.

In this problem $\mu$ is given by $\min \{M(z):-\infty<z<\infty\}$ which we denote by $\alpha$, and by strict convexity and $M(z) \rightarrow \infty$ there is a unique $z_{0}$ such that $\alpha=M\left(z_{0}\right)$. To see that $\mu=\alpha$, let $z(t)$ be a periodic trajectory of period $N \geqslant 1$. Then $\frac{1}{N} \int_{0}^{N}\left[\dot{z}(t)^{2}+M(z(t))\right] \mathrm{dt} \geqslant \frac{1}{N} \int_{0}^{N} M(z(t)) \mathrm{d} t$ and by the convexity of $M$ and the Jensen inequality (see Rudin [5], page 63),

$$
\frac{1}{N} \int_{0}^{N} M(z(t)) d t \geqslant M\left(\frac{1}{N} \int_{0}^{N} z(t) d t\right) \geqslant \alpha .
$$

Hence $\mu \geqslant \alpha$, while $\mu \leqslant \alpha$ follows from $\alpha=M\left(z_{0}\right)$. If $\mu=0$, then the problem is a minimization of finite integrals. If $\mu \neq 0$, still $\int_{0}^{\infty}\left[u^{2}+L(z)-\mu\right] d t$ can be made finite, so as an answer to the question raised in (1), the problem does make sense. Denote by $z_{T}(t)$ and $u_{T}(t)$ the unique optimal solution and optimal control in 
the interval $[0, T)$. It follows from the discussion in Proposition 5.3 that $z_{\infty}(t) \rightarrow$ $z_{0}$, and that $u_{\infty}(t) \rightarrow 0$. It is also possible to show that $z_{T}(T) \rightarrow z_{0}$ and in fact $z_{T}(t) \rightarrow z(t)$ as $T \rightarrow \infty$. The optimal modified cost for the finite intervals converges to the optimal modified cost for the infinite interval. These answer in the affirmative the question raised in (2).

\section{On the Compactness of $K$}

In this section we prove that in a certain special case one need not assume the existence of a compact set $K$ which includes all the programs. In this case we can prove the existence of a compact set $K$ such that for every sequence not included in it there is a sequence in $K$ which overtakes the former.

Thus from the optimality point of view we can confine ourselves to consider only sequences in $K$.

Theorem 8.1. Let $v: R^{n} \times R^{n} \rightarrow R^{1}$ be a lower semicontinuous function such that

$$
v(x, y) \rightarrow \infty \text { as }|x|+|y| \rightarrow \infty .
$$

Given a compact set $C \subset R^{n}$, there is a ball $B$ such that for every sequence $\left\{z_{i}\right\}_{i=0}^{\infty}$ not included in $B$, with $z_{0} \in C$, there exists a sequence $\left\{s_{i}\right\}_{i=0}^{\infty} \subset B$ with $s_{0}=z_{0}$ such that

$$
\sum_{i=0}^{N} v\left(s_{i}, s_{i+1}\right)<\sum_{i=0}^{N} v\left(z_{i}, z_{i+1}\right) \text { for all large } N \text {. }
$$

To prove the theorem we shall need the following:

Proposition 8.2. For a compact $C \subset R^{n}$ there are a ball $B_{0} \supset C$ and an integer $N_{0}$ such that for every sequence $\left\{z_{i}\right\}_{i=0}^{\infty}$ which contains $N_{0}$ consecutive members not belonging to $B_{0}$ there is a sequence $\left\{s_{i}\right\}_{i=0}^{\infty} \subset B_{0}$ so that (8.2) holds.

Proof. Let $t \in C$ and $\beta=v(t, t)$. Choose $B_{0}$ a ball large enough so that $B_{0} \supset C$ and $(x, y) \notin B_{0} \times B_{0} \Rightarrow v(x, y) \geqslant \beta+1$. Denote $a=\max _{(x, y) \in B_{0} \times B_{0}} v(x, y)$, and choose $N_{0}=[2(a-\beta)]+1$ (here $[x]$ is the integer part of $x$ ). Now if $\left\{z_{i}\right\}_{i=0}^{\infty}$ satisfies $z_{p+i} \notin B_{0}$ for $1 \leqslant i \leqslant N_{0}$ for some $p$, then there are two possibilities: Either $z_{p+i} \notin B_{0}$ for all $i \geqslant 1$, or there is a first integer $l$ so that $z_{p+l} \in B_{0}$. In the first possibility the costs for large $N$ grows at least as $(\beta+1) N$ so the sequence $\left\{s_{i}\right\}$ for which $s_{i}=t, i \geqslant p$ will satisfy (8.2). In the second possibility we have: $z_{p} \in B_{0}, \quad z_{p+i} \notin B_{0} \quad$ for $1 \leqslant i \leqslant l-1, \quad z_{p+l} \in B_{0}$ and $l>N_{0}$. Therefore 


$$
\begin{aligned}
& p+l-1 \\
& \sum_{i=p} v\left(z_{i}, z_{i+1}\right) \geqslant l(\beta+1) \text {. Define } \\
& s_{k}= \begin{cases}t & p<k<p+l \\
z_{k} & k \leqslant p \quad \text { or } k \geqslant p+l\end{cases}
\end{aligned}
$$

then

$$
\sum_{k=p}^{p+l-1} v\left(s_{k}, s_{k+1}\right) \leqslant 2 a+(l-2) \beta<l(\beta+1)
$$

and (8.2) holds.

Proof of the Theorem. Let $B_{0}, N_{0}$ be as asserted in Proposition 8.2. For each $2 \leqslant k \leqslant N_{0}$ there exists by (8.1) a ball $B_{k}$ such that $\left\{z_{0}, z_{1}, \ldots, z_{k}\right\} \not \subset B_{k}$ implies

$$
\sum_{i=0}^{k-1} v\left(z_{i}, z_{i+1}\right)>2|a|+\left(N_{0}-2\right)|\beta| \text {. }
$$

Any section of sequence of $k+1$ members $\left\{z_{p}, z_{p+1}, \ldots, z_{p+k}\right\}$ such that $z_{p} \in B_{0}$, $z_{p+k} \in B_{0},\left\{z_{p}, z_{p+1}, \ldots, z_{p+k}\right\} \not \subset B_{k}$ can be replaced by $\left\{z_{p}, t, \ldots, t, z_{p+k}\right\}$ and thus diminishing the costs for all large $N$ 's. Define $B=B_{0} \cup\left(\bigcup_{k=2}^{N_{0}} B_{k}\right)$. Any section of length $2 \leqslant k \leqslant N_{0}$ whose end points are in $B_{0}$ and which is not contained in $B$ is not contained in $B_{k}$ either and can be replaced by a better one which is in $B$. The same is true regarding sections longer than $N_{0}$ which are not contained in $B$, as indicated in Proposition 8.2. This concludes the proof.

\section{Acknowledgments}

I wish to express my deep gratitude to Professor Zvi Artstein for his constant advice and for fruitful, inspiring discussions. I also would like to thank an anonymous referee whose valuable comments much improved the quality of this paper.

\section{References}

1. Bellman R, Bucy R (1964) Asymptotic control theory. SIAM Control 2:11-18

2. Brock WA, Haurie A (1976) On existence of overtaking optimal trajectories over an infinite time horizon. Math Op Res 1:337-346

3. Gale D (1967) On optimal development in a multi-sector economy. Rev Econ Studies 34:1-19

4. Rockefellar RT (1979) Convex processes and Hamiltonian dynamical systems. In: Krein J (ed) Convex Analysis and Mathematical Economics. Lecture Notes in Economics and Mathematical Systems 168:122-136

5. Rudin W (1974) Real and Complex Analysis. McGraw-Hill Series in Higher Mathematics. New York, McGraw-Hill

6. von Weizsacker CC (1965) Existence of optimal programs of accumulation for an infinite horizon. Rev Econ Studies 32:85-104 